\title{
De la structure tarifaire à sa mise en application
}

\section{Urs Stoffel}

Dr, membre du Comité central de la FMH, responsable ad intérim du département Tarifs et conventions pour la médecine ambulatoire en Suisse

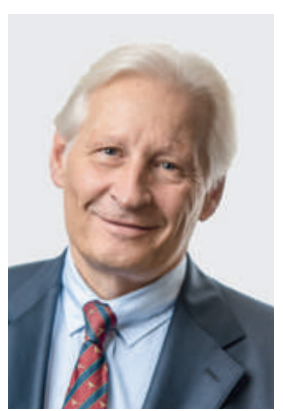

Le projet TARVISION, qui porte sur la révision de la structure tarifaire ambulatoire TARMED, est entré dans une phase décisive. Actuellement, plus de 150 personnes sont à l'œuvre au sein d'une vingtaine d'équipes spécialisées composées de représentants des partenaires en charge de la révision - la FMH, H+ et la CTM et des délégués tarifaires des sociétés de discipline médicale concernées par les différentes positions tarifaires.

$\mathrm{Au}$ vu du calendrier particulièrement ambitieux de la révision, l'interaction entre les différentes composantes du projet laisse peu de marge de manœuvre si l'on veut éviter de prendre du retard. Différents obstacles restent à franchir, pour lesquels la FMH et ses partenaires ont besoin de la précieuse collaboration et du savoir-faire des délégués tarifaires des sociétés de discipline.

Les règles et les paramètres nécessaires à l'application quotidienne d'un tarif à la prestation doivent faire l'objet de négociations et de conventions entre les partenaires tarifaires.

Jusqu'ici, le projet TARVISION s'est principalement concentré sur la révision de la structure tarifaire. Mais une structure tarifaire n'est pas encore un tarif pouvant être appliqué au quotidien. Pour cela, il faut fixer des règles et des paramètres qui doivent ensuite faire l'objet de négociations et de conventions entre les partenaires tarifaires, ce qui prend du temps. Comme le système de santé de notre pays de même que l'application de la LAMal relèvent essentiellement des cantons, il ne suffit pas que les partenaires tarifaires signent une convention-cadre nationale; il faut aussi des conventions d'adhésion cantonales. Il en va de même de la valeur du point tarifaire, négociée et fixée au plan cantonal. Enfin, après une révision aussi conséquente, il s'agit également de recalculer les valeurs de départ du point tarifaire.

Lors de l'introduction du TARMED en 2004, la convention-cadre nationale avait été négociée par la FMH et $\mathrm{H}+$ pour les fournisseurs de prestations, ainsi que par santésuisse et la CTM pour les répondants des coûts. Concernant les conventions d'adhésion cantonales, un groupe nommé «G7» avait vu le jour et négocié un modèle de convention avec santésuisse sur mandat des cantons. C'est également ce G7 qui avait été chargé de calculer la valeur de départ du point tarifaire de chaque canton en collaboration avec NewIndex, puis de la négocier avec santésuisse.

Aujourd'hui, la situation est beaucoup plus complexe. En effet, les répondants des coûts de la LAMal sont désormais représentés par deux entités indépendantes, santésuisse et curafutura. Dans ce contexte, plusieurs questions se posent: faut-il négocier la conventioncadre nationale - qui, rappelons-le, vise à uniformiser la structure tarifaire comme l'exige la LAMal - de manière séparée avec chacune de ces deux organisations faîtières? Ou celles-ci doivent-elles mettre sur pied une délégation commune? Que se passerait-il si aucune convention-cadre commune ne voyait le jour faute d'accord? Cette question se pose également pour les conventions d'adhésion cantonales: qui négocie avec qui sur quel mandat le modèle de convention d'adhésion cantonale? Enfin, la convention-cadre nationale devra encore être approuvée par le Conseil fédéral avec la nouvelle structure tarifaire, les conventions d'adhésion cantonales et les valeurs du point tarifaire par les gouvernements cantonaux. Tout cela exige une grande coordination mais surtout du temps!

Comme vous le voyez, chères et chers Collègues, de nombreuses questions sont encore en suspens et de

\section{L'interaction entre les différentes composantes} du projet laisse peu de marge de manœuvre si l'on veut éviter que la révision ne prenne du retard.

nombreux points restent à clarifier. C'est un travail de longue haleine qui nous attend ces prochaines semaines et ces prochains mois avant que la structure tarifaire révisée puisse être mise en œuvre et qu'un nouveau tarif susceptible d'être appliqué au quotidien voie le jour. 